\title{
Longitudinal Evaluation of Fatty Acid Metabolism in Normal and Spontaneously Hypertensive Rat Hearts with Dynamic MicroSPECT Imaging
}

\author{
Bryan W. Reutter, Ronald H. Huesman, Kathleen M. Brennan, Rostyslav Boutchko, \\ Stephen M. Hanrahan, and Grant T. Gullberg
}

Department of Radiotracer Development \& Imaging Technology, Lawrence Berkeley National Laboratory, One Cyclotron Road, Berkeley, CA 94720, USA

Correspondence should be addressed to Bryan W. Reutter, bwreutter@lbl.gov

Received 2 August 2010; Revised 15 September 2010; Accepted 16 September 2010

Academic Editor: Seigo Kinuya

Copyright (C) 2011 Bryan W. Reutter et al. This is an open access article distributed under the Creative Commons Attribution License, which permits unrestricted use, distribution, and reproduction in any medium, provided the original work is properly cited.

The goal of this project is to develop radionuclide molecular imaging technologies using a clinical pinhole SPECT/CT scanner to quantify changes in cardiac metabolism using the spontaneously hypertensive rat (SHR) as a model of hypertensive-related pathophysiology. This paper quantitatively compares fatty acid metabolism in hearts of SHR and Wistar-Kyoto normal rats as a function of age and thereby tracks physiological changes associated with the onset and progression of heart failure in the SHR model. The fatty acid analog, ${ }^{123}$ I-labeled BMIPP, was used in longitudinal metabolic pinhole SPECT imaging studies performed every seven months for 21 months. The uniqueness of this project is the development of techniques for estimating the blood input function from projection data acquired by a slowly rotating camera that is imaging fast circulation and the quantification of the kinetics of ${ }^{123}$ I-BMIPP by fitting compartmental models to the blood and tissue time-activity curves.

\section{Introduction}

Hypertrophic cardiomyopathy is a condition in which the heart muscle becomes thick, forcing the heart to work harder to pump blood. Under normal conditions the heart uses glucose $(\sim 30 \%)$, fatty acids $(\sim 60 \%)$, and lactate $(\sim 10 \%)$ as primary energy sources, in addition to amino acids and ketone bodies [1-3]. In the case of cardiac hypertrophy, however, there is an increase in cardiac mass and a switch to a reliance on glucose metabolism. To be able to detect and interpret the early onset of this change, there is the need to develop methodology for sensitive predictors for early detection, prognosis and to follow the response to therapy for hypertrophic cardiomyopathy. In clinical settings, the abnormalities of fatty acid metabolism in hypertrophic cardiomyopathy can be recognized by the decreased uptake in single-photon emission computed tomography (SPECT) images [4]. It has also been demonstrated that compartmental analysis and dynamic SPECT imaging make it possible to detect abnormalities of fatty acid utilization earlier than SPECT imaging, with the potential to provide an even earlier prediction of the onset of cardiac hypertrophy [5]. There is a need to develop technology for imaging small animal models on clinical SPECT systems that could easily be translated to the clinic for diagnosis and management of patients with cardiac hypertrophy. However, the challenge is to perform compartmental analysis of dynamic studies in small animals with pinhole SPECT using slowly rotating gantries (slow camera rotation with $1 \mathrm{~s}$ per view) when the recirculation time in the animals is $6-8$ s. This study was designed to follow the changes in fatty acid metabolism in the left ventricular myocardium associated with the progression of hypertrophy in the spontaneously hypertensive rat (SHR) and, in so doing, develop methodology for data acquisition and data processing techniques of pinhole SPECT acquired data [611]. The goal of this project is to develop radionuclide molecular imaging technologies using a clinical dual-modality pinhole SPECT/X-ray computed tomography (CT) scanner 


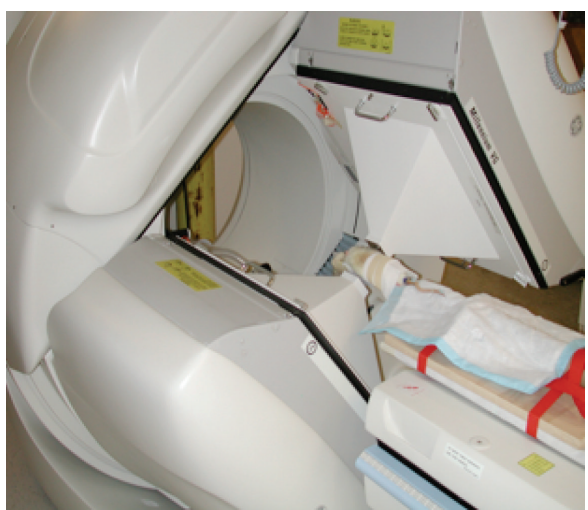

(a)

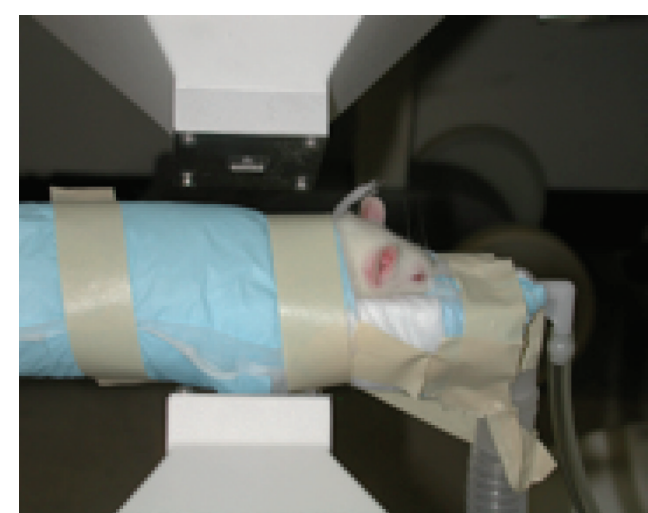

(b)

FIGURE 1: Clinical dual-detector SPECT/CT scanner with custom pinhole collimators used for quantitative dynamic imaging of fatty acid metabolism in the rat heart.

to quantify the changes in metabolism in the heart using the SHR as a model of hypertensive-related pathophysiology (Figure 1).

The SHR (Okamoto and Aoki strain) has hypertension associated with generalized dyslipidemia and insulin resistance. It has been noted that the SHR model has a defective gene (CD36) on chromosome 4 [12]. The gene in the SHR results in a defective fatty acid enzyme (translocase), which functions in long-chain fatty acid transport into the cell. This compromises tissue utilization of fatty acid and increases the basal glucose metabolism and hyperinsulinemia.

Mechanisms involved in the progression of heart failure are believed to be, in part, related to alteration in myocardial energy metabolism [13-15]. Plasma levels of glucose, fatty acids, and lactate determine which of these substrates are oxidized [16]. In the failing heart, energy substrate utilization changes from fatty acid oxidation to one of glycolysis utilization $[17,18]$. In the case of pressure overload, there is an increased reliance on carbohydrate oxidation in an attempt to maintain contractile function. The myocardial extraction and retention of fatty acids are impaired in the advanced stage of heart failure [19]. The fatty acid analog, $\beta$-methyl-p-[ $\left.{ }^{123} \mathrm{I}\right]$-iodophenyl-pentadecanoic acid $\left({ }^{123} \mathrm{I}\right.$ BMIPP), currently being evaluated in human studies to study the progression of heart failure, has been used to image the metabolism of fatty acids $[1,20]$. Imaging with ${ }^{123} \mathrm{I}-$ BMIPP in a canine has shown that myocardial fatty acid oxidation begins to be inhibited and that washout of ${ }^{123} \mathrm{I}$ BMIPP increases in the compensated stage of left ventricular dysfunction. Human, canine, and rodent studies show that in late-stage heart failure there is downregulation of myocardial fatty acid oxidation and accelerated glucose oxidation [2123]. The reduction in fatty acid oxidation is not caused by changes in fatty acid availability in the blood [24]. The time course and the molecular mechanisms for this switch in substrate oxidation are not well understood $[25,26]$.

This paper quantitatively compares fatty acid metabolism in the hearts of SHR and Wistar-Kyoto (WKY) normal rats as a function of age, and thereby tracks physiological changes associated with the onset and progression of heart failure in the SHR model. ${ }^{123}$ I-BMIPP was used in longitudinal metabolic pinhole SPECT imaging studies performed every seven months for 21 months. The uniqueness of this project is the development of techniques for estimating the blood input function from projection data acquired by a slowlyrotating camera imaging fast circulation in a rat, and the quantification of the kinetics of ${ }^{123}$ I-BMIPP by fitting compartmental models to the blood input function and tissue uptake/washout time-activity curves (TACs).

In previous work, we addressed issues associated with reconstructing dynamic data acquired with use of a slowlyrotating camera [27-29]. The work presented here also addresses quantitative effects of limited spatial resolution in dynamic pinhole SPECT that result in underestimation of the metabolic rate of ${ }^{123}$ I-BMIPP in the rat myocardium. In particular, the partial volume effect blurs activity between the left ventricular blood pool and surrounding myocardial tissue and decreases contrast between the blood input and tissue uptake TACs $[11,30,31]$. Standard compartmental modeling straightforwardly accounts for the spillover of blood activity into tissue volumes [32]. However, accounting for the spillover of tissue activity into blood volumes is more problematic, and is a focus of the work presented here. Results are presented for imaging studies performed on two SHRs and two WKY normal rats at three ages.

This paper is organized as follows. Section 2 begins by describing the data acquisition protocol for acquiring dynamic ${ }^{123}$ I-BMIPP data in a rat with use of a slowlyrotating dual-detector pinhole SPECT system. This section then provides overviews of our multiresolution methods for fully $3 \mathrm{D}$ reconstruction of a late static SPECT image (to determine the spatial locations for the left ventricular blood pool and myocardial tissue) and for fully 4-D reconstruction of an early dynamic SPECT image represented by 4-D splines that are piecewise constant in space and piecewise quadratic in time. Section 2 concludes with details of our fully 4-D penalized least-squares reconstruction algorithm that uses a smooth 4-D image prior, as well as our methods for jointly estimating the blood input function and fatty acid metabolism from the reconstructed dynamic SPECT 


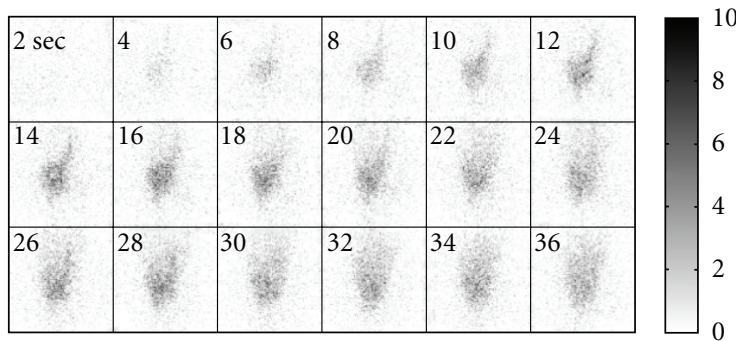

(a) WKY normal A, 14 months

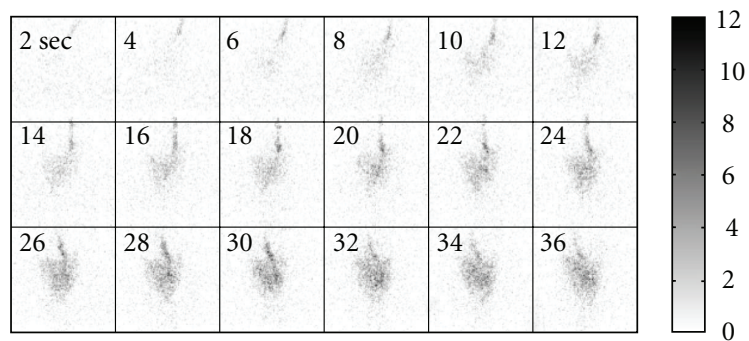

(b) SHR A, 14 months

FIGURE 2: Dynamic cardiac ${ }^{123}$ I-BMIPP pinhole SPECT projection data acquired by one detector head during the interval 2-36 s for (a) a WKY normal rat and (b) an SHR. These early time frames show the arrival of the injected bolus at the heart, followed by initial uptake in the myocardium. The maximum numbers of counts in a detector bin are 10 and 12 for the WKY normal rat and SHR, respectively.

image. Section 3 presents results obtained with use of these algorithms applied to dynamic SPECT imaging studies performed on two SHR and two WKY normal rats when the rats were age 7, 14, and 21 months (one SHR died before 21 months). The paper concludes with a discussion of the results and future work in Section 4.

\section{Materials and Methods}

2.1. Pinhole SPECT System Modeling and Data Acquisition. With use of methods described in $[8,9]$, dynamic cardiac pinhole SPECT projection data and pinhole geometric calibration data were acquired with slow gantry rotation on a dual-detector GE Millennium VG Hawkeye SPECT/CT scanner equipped with custom tungsten pinhole collimators having a $1.5 \mathrm{~mm}$ by $2 \mathrm{~mm}$ retangular aperture (Figure 1 ). The $\mathrm{NaI}(\mathrm{Tl})$ crystal in each detector has an area of $540 \mathrm{~mm}$ by $400 \mathrm{~mm}$ and is $9.5 \mathrm{~mm}$ thick. At an energy of $140 \mathrm{keV}$, the intrinsic spatial resolution is $3.9 \mathrm{~mm}$ and the energy resolution is $9.8 \%$.

In the geometric configuration used for imaging rats (Figure 1), the pinhole collimators magnify the center of the field of view by a factor of about 4.8 on the faces of the large detectors. In a phantom study that we performed in this configuration $[8,9]$, the system could easily resolve the smallest features of the micro-Jaszczak phantom, which are $1.2 \mathrm{~mm}$ "cold" rod sources separated by $1.2 \mathrm{~mm}$ in a radioactive background [33]. Collimator response was modeled via ray tracing and excluded the effects of collimator penetration. The system model also excluded the effects of attenuation and scatter; however, we are currently studying these effects via Monte Carlo simulation in a separate investigation $[34,35]$.

${ }^{123}$ I-BMIPP was obtained from Molecular Insight Pharmaceuticals. All imaging studies were performed in accordance with an Institutional Animal Care and Use Committee (IACUC) approved protocol. Rats were anesthetized throughout the entire procedure with use of $2-2.5 \%$ isoflurane inhalation anesthesia. A slow (10-30s) injection of about $4 \mathrm{mCi}(150 \mathrm{MBq})$ of ${ }^{123}$ I-BMIPP, via an IV catheter placed in the tail vein, was performed shortly after the dynamic data acquisition began. Data were acquired for
$60 \mathrm{~min}$ in 1-s time frames with an angular step of 4 degrees per frame (Figure 2). For the biodistributions associated with these studies, overall system sensitivity was about $2000 \mathrm{cps} / \mathrm{mCi}$ ( $55 \mathrm{cps} / \mathrm{MBq}$ ). The energy of the primary photopeak for ${ }^{123} \mathrm{I}$ is $159 \mathrm{keV}$.

2.2. Multiresolution Fully 3D Late Static SPECT Image Reconstruction. To determine spatial locations for the left ventricular blood pool and myocardial tissue, late data acquired 1.5-60 min after injection were summed and a static image was reconstructed with use of a 3D version of the penalized least-squares image reconstruction that we describe in Section 2.4. The late static spatial distribution of ${ }^{123}$ I-BMIPP was modeled with use of 3D multiresolution spatial B-splines that were piecewise constant. The 3D spatial splines were organized on a $20 \times 20 \times 203 \mathrm{D}$ grid that provided uniform sampling of $3.2 \mathrm{~mm}$ in each dimension. Inside the volume containing the heart, a $6 \times 6 \times 6$ neighborhood of these lower-resolution splines was replaced by a $12 \times 12 \times 12$ neighborhood of higher-resolution splines that provided uniform sampling of $1.6 \mathrm{~mm}$.

\subsection{Multiresolution Fully 4-D Early Dynamic SPECT Image} Reconstruction. The time-varying spatial distribution of ${ }^{123} \mathrm{I}-$ BMIPP was modeled with use of 4-D multiresolution Bsplines that were piecewise constant in space and piecewise quadratic in time. The $4-\mathrm{D}$ splines were spatially organized on a $10 \times 10 \times 103 \mathrm{D}$ grid that provided uniform sampling of $6.4 \mathrm{~mm}$ in each dimension. Inside the volume containing the heart, a $3 \times 3 \times 3$ neighborhood of these lower-resolution splines was replaced by a $12 \times 12 \times 12$ neighborhood of higher-resolution splines that provided uniform sampling of $1.6 \mathrm{~mm}$. The 4 -D splines were temporally organized on a $1 \mathrm{D}$ grid that provided nonuniform sampling intervals of $0-2.4$, 2.4-9.4, 9.4-30, and 30-90 s during the first gantry rotation (Figure 3).

With use of the fully 4-D algorithm for penalized leastsquares image reconstruction that we describe in Section 2.4, B-spline TACs for the multiresolution voxels were estimated directly from the dynamic pinhole SPECT projection data. This yielded estimates of temporal B-spline coefficients 


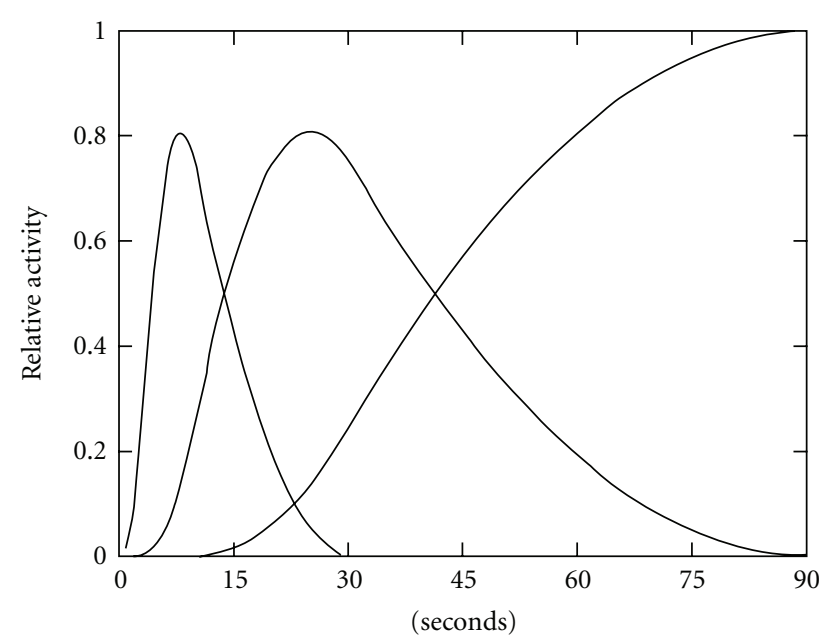

Figure 3: Piecewise quadratic temporal B-spline basis functions used to reconstruct dynamic data acquired during the first gantry rotation.

$\left\{\hat{a}_{m n} ; m=1, \ldots, M ; n=1, \ldots, N\right\}$, where $M$ is the number of spatial voxels and $N$ is the number of temporal B-spline basis functions. The estimated TAC for the $m$ th voxel is

$$
\widehat{A}^{m}(t)=\sum_{n=1}^{N} \hat{a}_{m n} V^{n}(t),
$$

where $V^{n}(t)$ are temporal B-spline basis functions (Figure 3).

2.4. Fully 4-D Penalized Least-Squares Reconstruction Algorithm with a Smooth 4-D Image Prior. A dynamic SPECT projection data model that relates detected events to a 4D spatiotemporal B-spline representation of a time-varying radiotracer distribution can be written as

$$
\mathbf{p}=\mathbf{F a},
$$

where $\mathbf{p}$ is an $I$-element column vector of modeled dynamic projection data values, $\mathbf{F}$ is an $I \times(M N)$ system matrix, $\mathbf{a}$ is an (MN)-element column vector of B-spline coefficients, and $I$ is the total number of projection measurements acquired by the SPECT detectors. The system matrix $\mathbf{F}$ incorporates the spline model for time variation of the radiotracer distribution, as well as physical effects such as collimator response that affect detection of gamma rays emitted by the radiotracer distribution.

At the outset, the least-squares criterion to be minimized, $\chi^{2}$, is simply the sum of squared differences between the measured projections, $\mathbf{p}^{*}$, and the modeled projections

$$
\chi^{2}=\left(\mathbf{p}^{*}-\mathbf{F a}\right)^{\mathrm{T}}\left(\mathbf{p}^{*}-\mathbf{F a}\right)
$$

where the superscript " $T$ " denotes the matrix transpose. Minimizing the criterion $\chi^{2}$ yields an estimate, $\hat{\mathbf{a}}$, of coefficients for the 4-D B-spline basis functions that represent the time-varying radiotracer distribution

$$
\hat{\mathbf{a}}=\left(\mathbf{F}^{\mathrm{T}} \mathbf{F}\right)^{-1} \mathbf{F}^{\mathrm{T}} \mathbf{p}^{*}
$$

The corresponding minimum value for the criterion $\chi^{2}$ is

$$
\chi_{\min }^{2}=\left(\mathbf{p}^{*}-\mathbf{F} \hat{\mathbf{a}}\right)^{\mathrm{T}}\left(\mathbf{p}^{*}-\mathbf{F} \hat{\mathbf{a}}\right)
$$

To reduce noise, we now wish to add a penalty term to the criterion $\chi^{2}$ that encourages the reconstructed image to be smooth in both space and time. Insight into what a reasonable penalty term might be can be obtained by expressing $\chi^{2}$ in terms of its minimum value

$$
\begin{aligned}
\chi^{2} & =\left(\mathbf{p}^{*}-\mathbf{F a}\right)^{\mathrm{T}}\left(\mathbf{p}^{*}-\mathbf{F a}\right) \\
& =\left[\left(\mathbf{p}^{*}-\mathbf{F} \hat{\mathbf{a}}\right)-\mathbf{F}(\mathbf{a}-\hat{\mathbf{a}})\right]^{\mathrm{T}}\left[\left(\mathbf{p}^{*}-\mathbf{F} \hat{\mathbf{a}}\right)-\mathbf{F}(\mathbf{a}-\hat{\mathbf{a}})\right] \\
& =\chi_{\min }^{2}-2\left(\mathbf{p}^{*}-\mathbf{F} \hat{\mathbf{a}}\right)^{\mathrm{T}} \mathbf{F}(\mathbf{a}-\hat{\mathbf{a}})+(\mathbf{a}-\hat{\mathbf{a}})^{\mathrm{T}} \mathbf{F}^{\mathrm{T}} \mathbf{F}(\mathbf{a}-\hat{\mathbf{a}}) \\
& =\chi_{\min }^{2}+(\mathbf{a}-\hat{\mathbf{a}})^{\mathrm{T}} \mathbf{F}^{\mathrm{T}} \mathbf{F}(\mathbf{a}-\hat{\mathbf{a}})
\end{aligned}
$$

Note that the term that is linear with respect to $(\mathbf{a}-\hat{\mathbf{a}})$ vanishes because the model error $\mathbf{p}^{*}-\mathbf{F}$ a lies in the null space of the backprojection operator $\mathbf{F}^{\mathrm{T}}$. Inspecting (6), one sees that differences from the least-squares solution $\hat{\mathbf{a}}$ are penalized by the term $(\mathbf{a}-\hat{\mathbf{a}})^{\mathrm{T}} \mathbf{F}^{\mathrm{T}} \mathbf{F}(\mathbf{a}-\hat{\mathbf{a}})$.

To mimic this effect for purposes of reducing noise, we propose to add a penalty term that resembles (a $\boldsymbol{\alpha})^{\mathrm{T}} \mathbf{F}^{\mathrm{T}} \mathbf{F}(\mathbf{a}-\boldsymbol{\alpha})$, where $\boldsymbol{\alpha}$ is a smooth 4-D image prior obtained by normalizing a simple backprojection of the measured projections

$$
\boldsymbol{\alpha}=\left(\mathbf{F}^{\mathrm{T}} \mathbf{p}^{*}\right) \cdot /\left(\mathbf{F}^{\mathrm{T}} \mathbf{F}[\mathbf{1}]\right)
$$

where the operator "./" denotes pointwise division of elements in the left operand by the corresponding elements in the right operand and "[1]" denotes an $(M N)$-element column vector of ones. Note that normalization by $\mathbf{F}^{\mathrm{T}} \mathbf{F}[\mathbf{1}]$ ensures that backprojecting the noiseless projections of a constant image yields the original constant image. Note also that the image prior $\boldsymbol{\alpha}$ has the desirable physiologic property of being nonnegative-thus, the reconstructed image is encouraged to have nonnegative 4-D B-spline coefficients.

The penalty term that we propose to use is

$$
\sum_{i=1}^{I} \sum_{m=1}^{M} \sum_{n=1}^{N}\left[F_{i}^{m n}\left(a_{m n}-\alpha_{m n}\right)\right]^{2}
$$

where $F_{i}^{m n}$ is the $[i, m+(n-1) M]$ th element of the system matrix F, $a_{m n}$ is the $[m+(n-1) M]$ th element of the spline coefficient vector $\mathbf{a}$, and $\alpha_{m n}$ is the $[m+(n-1) M]$ th element of the smooth image prior $\boldsymbol{\alpha}$. For the resulting negatively correlated, zero-mean elements in the vector $(\mathbf{a}-\boldsymbol{\alpha}),(8)$ imposes a penalty that is greater than the penalty imposed by $(\mathbf{a}-\boldsymbol{\alpha})^{\mathrm{T}} \mathbf{F}^{\mathrm{T}} \mathbf{F}(\mathbf{a}-\boldsymbol{\alpha})$, and is more effective at suppressing "checkerboard" noise patterns. Note that the latter penalty can be expressed in summation notation as

$$
\sum_{i=1}^{I}\left[\sum_{m=1}^{M} \sum_{n=1}^{N} F_{i}^{m n}\left(a_{m n}-\alpha_{m n}\right)\right]^{2}
$$




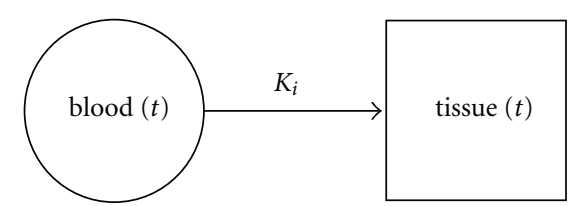

Figure 4: One-tissue-compartment model used for quantifying fatty acid metabolism during the first $90 \mathrm{~s}$ after injection of ${ }^{123} \mathrm{I}-$ BMIPP.

In general, the penalties (8) or (9) may be scaled by a smoothing parameter $\beta$. The penalized least-squares criterion that we minimized for the work presented here is

$$
\begin{aligned}
\Psi^{2}= & \sum_{i=1}^{I}\left[p_{i}^{*}-\sum_{m=1}^{M} \sum_{n=1}^{N} F_{i}^{m n} a_{m n}\right]^{2} \\
& +\beta \sum_{i=1}^{I} \sum_{m=1}^{M} \sum_{n=1}^{N}\left[F_{i}^{m n}\left(a_{m n}-\alpha_{m n}\right)\right]^{2},
\end{aligned}
$$

where the first term on the right-hand side is the leastsquares criterion (3) expressed in summation notation and the second term is the scaled penalty (8). The criterion $\Psi^{2}$ is minimized by the following estimate, $\hat{\mathbf{a}}$, of coefficients for the 4-D B-spline basis functions that represent the time-varying radiotracer distribution

$$
\widehat{\mathbf{a}}=\left[(\mathbf{1}+\beta \mathbf{I}) \cdot \mathbf{F}^{\mathrm{T}} \mathbf{F}\right]^{-1}\left[\mathbf{F}^{\mathrm{T}} \mathbf{p}^{*}+\beta \operatorname{diag}\left(\mathbf{F}^{\mathrm{T}} \mathbf{F}\right) \cdot \boldsymbol{\alpha}\right],
$$

where "1" is an $(M N) \times(M N)$ matrix of ones, $\mathbf{I}$ is an $(M N) \times(M N)$ identity matrix, "." denotes the Hadamard product (i.e., pointwise multiplication of elements in the left operand by the corresponding elements in the right operand), and $\operatorname{diag}\left(\mathbf{F}^{\mathrm{T}} \mathbf{F}\right)$ is an $(M N)$-element column vector whose $[m+(n-1) M]$ th element is the $[m+(n-1) M]$ th diagonal element of $\mathbf{F}^{\mathrm{T}} \mathbf{F}$.

Note that when the smoothing parameter $\beta$ is zero, (11) simplifies to (4); whereas, the image estimate $\hat{\mathbf{a}}$ approaches the smooth image prior $\alpha$ as $\beta$ approaches infinity. By virtue of (6), a reasonable value is $\beta=1$, which was used for the work presented here.

2.5. Joint Estimation of Blood Input and Fatty Acid Metabolism. To obtain a quantitative estimate of the metabolic rate of ${ }^{123}$ I-BMIPP in the myocardium, a one-tissue-compartment model (Figure 4) is fitted to TACs for higher-resolution voxels in a $7 \times 7 \times 7$ neighborhood centered on the blood pool. Early myocardial tissue uptake is modeled with a single, irreversible compartment

$$
\operatorname{tissue}(t)=K_{i} \cdot \int_{0}^{t} \operatorname{blood}(\tau) d \tau
$$

where $K_{i}$ is the metabolic rate of ${ }^{123}$ I-BMIPP. Each voxel is modeled as a mixture of blood input and tissue uptake, taking into account partial volume effects

$$
\begin{aligned}
\operatorname{voxel}(t)= & {\left[f_{v} \cdot \operatorname{blood}(t)\right] } \\
& +\left[\left(1-f_{v}\right) K_{i} \cdot \int_{0}^{t} \operatorname{blood}(\tau) d \tau\right],
\end{aligned}
$$

where $f_{v}$ is the fraction of vasculature in the tissue and also incorporates the effect of spillover from the blood pool to surrounding tissue voxels. For blood voxels, the factor $(1-$ $\left.f_{v}\right) K_{i}$ incorporates the effect of spillover from surrounding tissue voxels.

Because of tissue spillover, there is no reconstructed voxel that contains a pure blood TAC; thus, the blood input function is assumed to be unknown and is modeled by a Bspline TAC

$$
B(t)=\sum_{n=1}^{N} b_{n} V^{n}(t)
$$

The temporal B-spline coefficients $\left\{b_{n} ; n=1, \ldots, N\right\}$ are jointly estimated with compartmental model parameters $\left\{\left(f_{v}^{m}, k^{m}\right) ; m \in \Omega\right\}$ for each voxel by minimizing the following least-squares criterion:

$$
\sum_{m \in \Omega} \int_{0}^{T}\left\{\widehat{A}^{m}(t)-\left[f_{v}^{m} B(t)+k^{m} \int_{0}^{t} B(\tau) d \tau\right]\right\}^{2} d t,
$$

where $\Omega$ denotes the $7 \times 7 \times 7$ neighborhood of voxels centered on the blood pool, $T=90 \mathrm{~s}$, and $k^{m}=\left(1-f_{v}^{m}\right) K_{i}^{m}$. Thus, there is a total of $\left(2 \cdot 7^{3}\right)+3=689$ parameters to jointly estimate (i.e., two compartmental model parameters $f_{v}^{m}$ and $k^{m}$ for each of the 343 voxels in the neighborhood $\Omega$, and three blood curve B-spline coefficients $b_{1}, b_{2}$, and $b_{3}$ ).

The minimization proceeds by first initializing $B(t)$ to the TAC for the voxel at the center of the neighborhood $\Omega$. Then, optimal values for the B-spline coefficients $\left\{b_{n} ; n=\right.$ $1, \ldots, N\}$ for $B(t)$ are found with use of an iterative search algorithm. Note that one does not need to search explicitly for optimal values for the conditionally linear compartmental model parameters $\left\{\left(f_{v}^{m}, k^{m}\right) ; m \in \Omega\right\}$, as there are unique optimal values for these parameters given $B(t)[36,37]$.

Values for $K_{i}$ reported in the results are based on the final estimate for $B(t)$ and the average of TACs for 12 myocardial tissue voxels identified in static $3 \mathrm{D}$ images reconstructed from summed late data acquired 1.5-60 min after injection (Figure 5).

\section{Results}

In the late 3D static images (Figure 5), more trapping of ${ }^{123} \mathrm{I}-$ BMIPP is evident in the WKY normal hearts (top two rows), compared to the SHR hearts (bottom two rows). These static images have been normalized to one another by normalizing by the injected dose per unit body weight. Table 1 lists the body weight of each rat for each study.

For the early 4-D dynamic images, the use of nonuniform time sampling with splines that varied quadratically in time, 

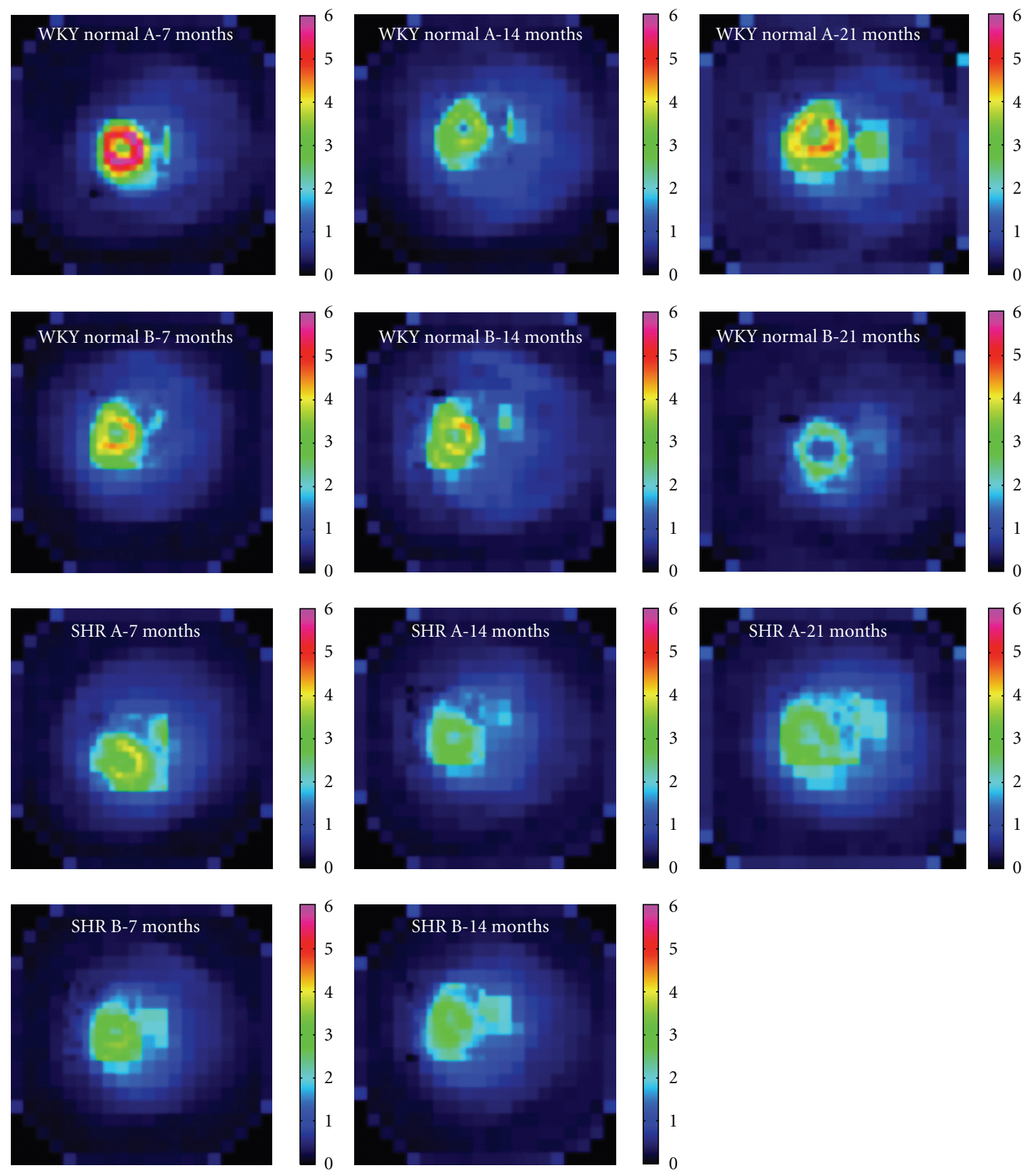

FIGURE 5: Typically, more trapping of ${ }^{123}$ I-BMIPP is evident in late 3D static images of the WKY normal hearts (top two rows), compared to the SHR hearts (bottom two rows). Trapping also tends to decrease with age (left column, 7 months; middle column, 14 months; right column, 21 months). SHR B died of congestive heart failure before 21 months. These static images have been normalized to one another by normalizing by the injected dose per unit body weight.

along with the use of a smooth 4-D image prior, yielded smooth time-activity curves that captured the relatively fast rise and fall of ${ }^{123} \mathrm{I}$-BMIPP in the left ventricular blood pool, as well as the uptake and initial trapping of the radiotracer in the left ventricular myocardium. Figure 6 shows time-activity curves for the spillover-corrected blood input function and myocardial uptake (triangles and circles, resp.), as well as the compartmental model fit (solid line) to the myocardial uptake curve, for each study.

The spillover of tissue activity into the left ventricular blood pool averaged $19 \pm 10 \%$ across all 11 studies. Tissue spillover correction compensated for partial volume effects and improved the contrast between the blood input and myocardial uptake curves for all studies and visually 


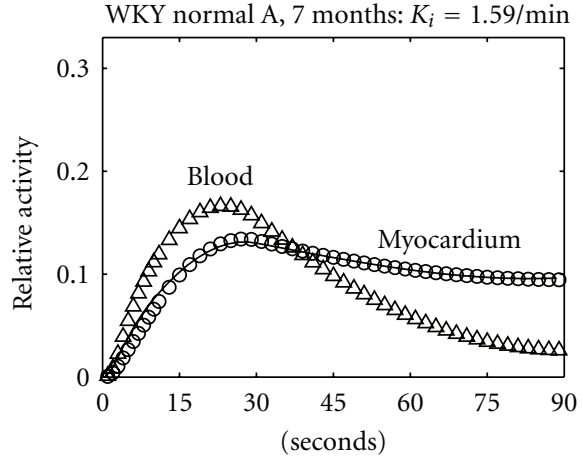

WKY normal B, 7 months: $K_{i}=0.83 / \mathrm{min}$

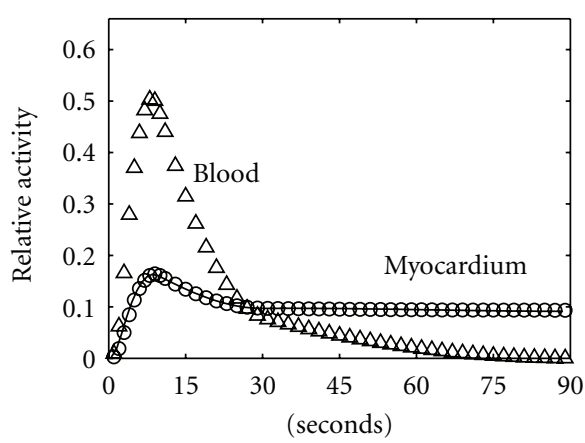

SHR A, 7 months: $K_{i}=1.12 / \mathrm{min}$

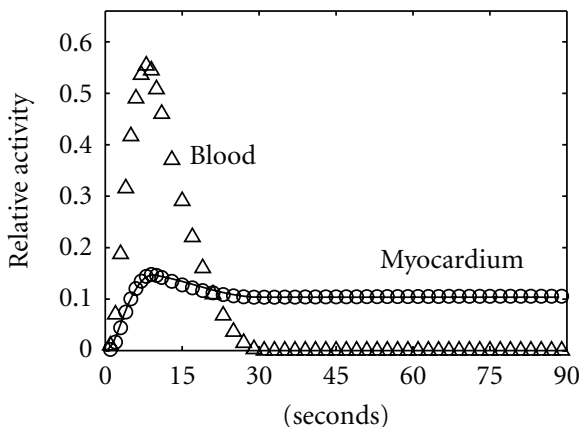

SHR B, 7 months: $K_{i}=2.15 / \mathrm{min}$

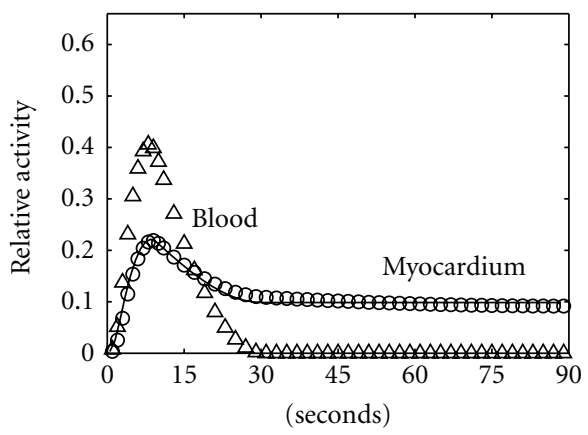

$\begin{array}{ll}\Delta & \text { TAC estimate } \\ \circ & \text { TAC estimate }\end{array}$

- Model fit

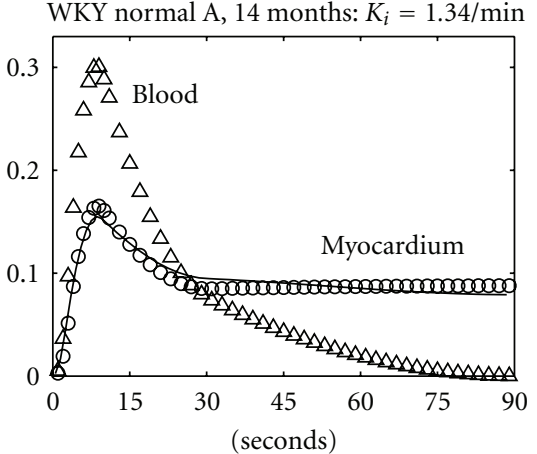

WKY normal B, 14 months: $K_{i}=1.47 / \mathrm{min}$

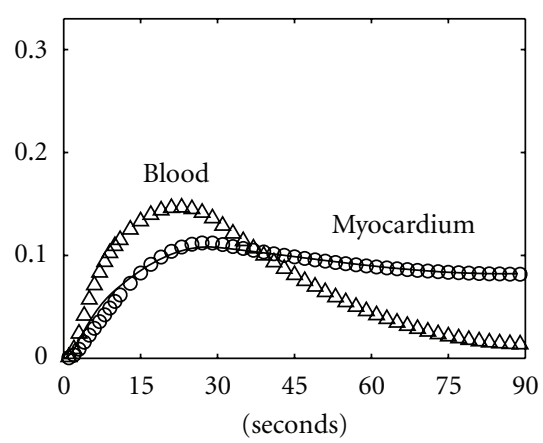

SHR A, 14 months: $K_{i}=0.54 / \mathrm{min}$

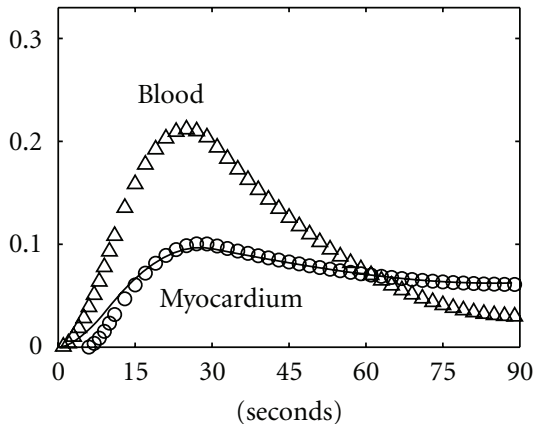

SHR B, 14 months: $K_{i}=0.62 / \mathrm{min}$

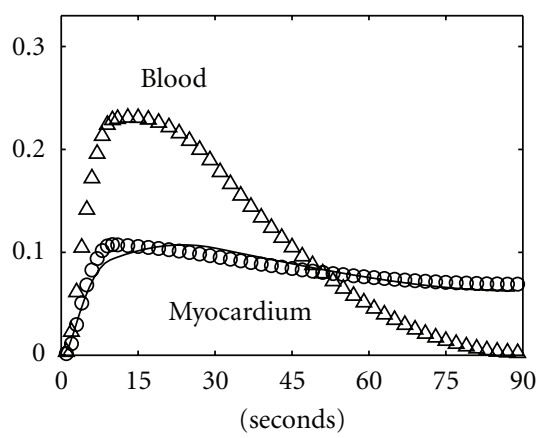

$\begin{array}{ll}\Delta & \text { TAC estimate } \\ \circ & \text { TAC estimate }\end{array}$

- TAC estim

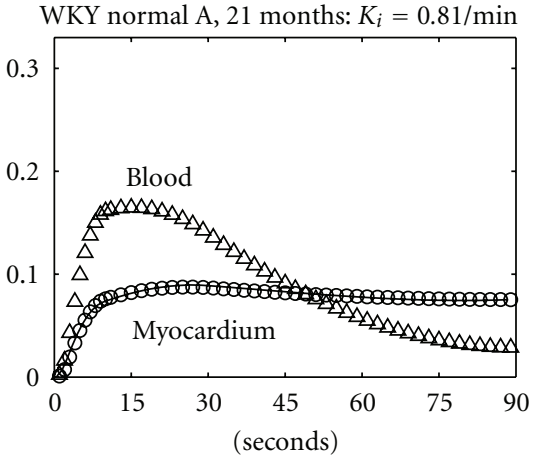

WKY normal B, 21 months: $K_{i}=0.41 / \mathrm{min}$

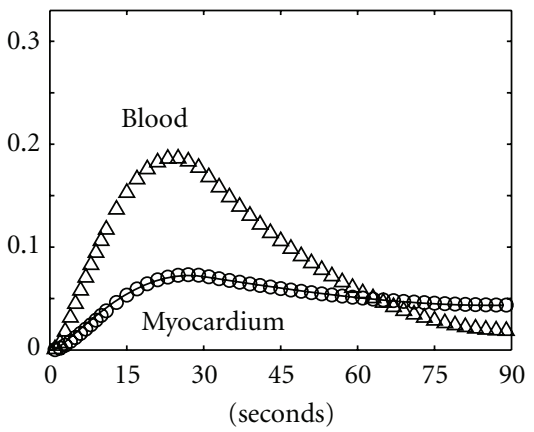

SHR A, 21 months: $K_{i}=0.5 / \mathrm{min}$

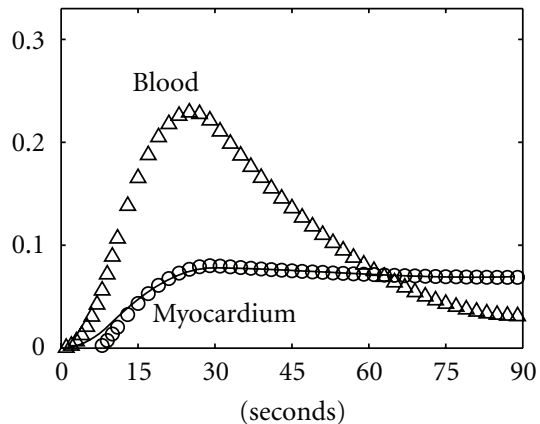

FIGURE 6: Time-activity curves for the WKY normal rats (top two rows) and the SHRs (bottom two rows) capture quantitative differences between their spillover-corrected blood inputs and myocardial uptakes (triangles and circles, resp.). Compartmental models (solid lines) provide good fits to the myocardial uptake curves. Left column, 7 months; middle column, 14 months; right column, 21 months. SHR B died of congestive heart failure before 21 months. 


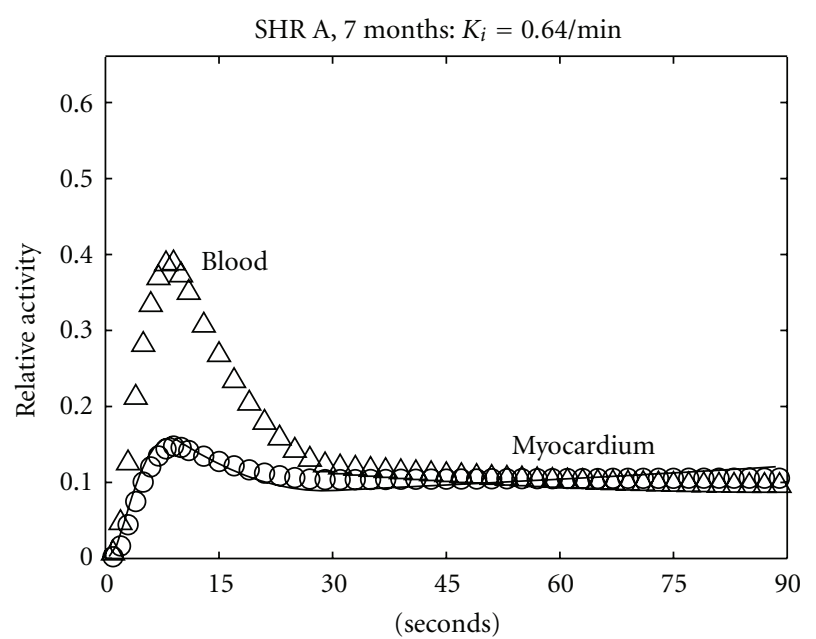

$$
\begin{array}{ll}
\triangle & \text { TAC estimate } \\
\bigcirc & \text { TAC estimate } \\
& \text { Model fit }
\end{array}
$$

(a) Blood curve not corrected for spillover from myocardium
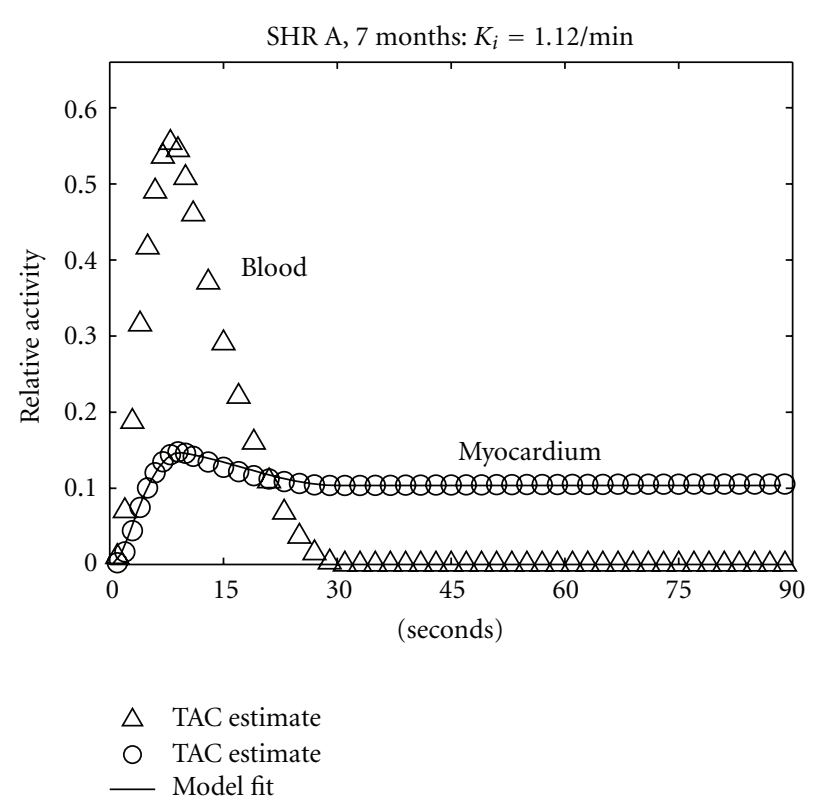

(b) Result of tissue spillover correction

FIgURE 7: Time-activity curves for SHR A at 7 months estimated (a) without and (b) with tissue spillover correction for the blood curve. Spillover correction improves contrast between the blood input and myocardial uptake (triangles and circles, resp.), improves the fit of the compartmental model (solid line), and yields a metabolic rate estimate $\left(K_{i}\right)$ that nearly doubles, from $0.64 \mathrm{~min}^{-1}$ to $1.12 \mathrm{~min}^{-1}$.

\begin{tabular}{|c|c|c|c|}
\hline & \multicolumn{3}{|c|}{ Body weight } \\
\hline & 7 Months & 14 Months & 21 Months \\
\hline WKY normal A & $420 \mathrm{~g}$ & $482 \mathrm{~g}$ & $545 \mathrm{~g}$ \\
\hline WKY normal B & $432 \mathrm{~g}$ & $494 \mathrm{~g}$ & $564 \mathrm{~g}$ \\
\hline SHR A & $430 \mathrm{~g}$ & $465 \mathrm{~g}$ & $416 \mathrm{~g}$ \\
\hline SHR B & $403 \mathrm{~g}$ & $446 \mathrm{~g}$ & - \\
\hline
\end{tabular}

TABLE 1: Body weight of each rat for each study. SHR B died of congestive heart failure before 21 months.

improved the fit of the compartmental model for some studies (Figure 7). Metabolic rate estimates $\left(K_{i}\right)$ increased by an average of $72 \pm 45 \%$ across all 11 studies, compared to estimates obtained without spillover correction.

Estimates of $K_{i}$ obtained from corrected blood curves are plotted as a function of age for all 11 studies in Figure 8. The general decline with age is what one expects. Slower rates of fatty acid metabolism in the SHRs at 14 months, compared to the WKY normal rats, is also expected as the SHR hearts switch to a reliance on glycolysis as the primary pathway for energy production during the development of heart failure. SHR B died of congestive heart failure before 21 months.

\section{Discussion}

We showed that it is potentially feasible to estimate the blood and myocardial tissue time-activity curves in rat models from projection measurements for dynamic data acquired with slow camera rotation of $1 \mathrm{~s}$ per projection, even when recirculation times are on the order of $6-8 \mathrm{~s}$. The tissue

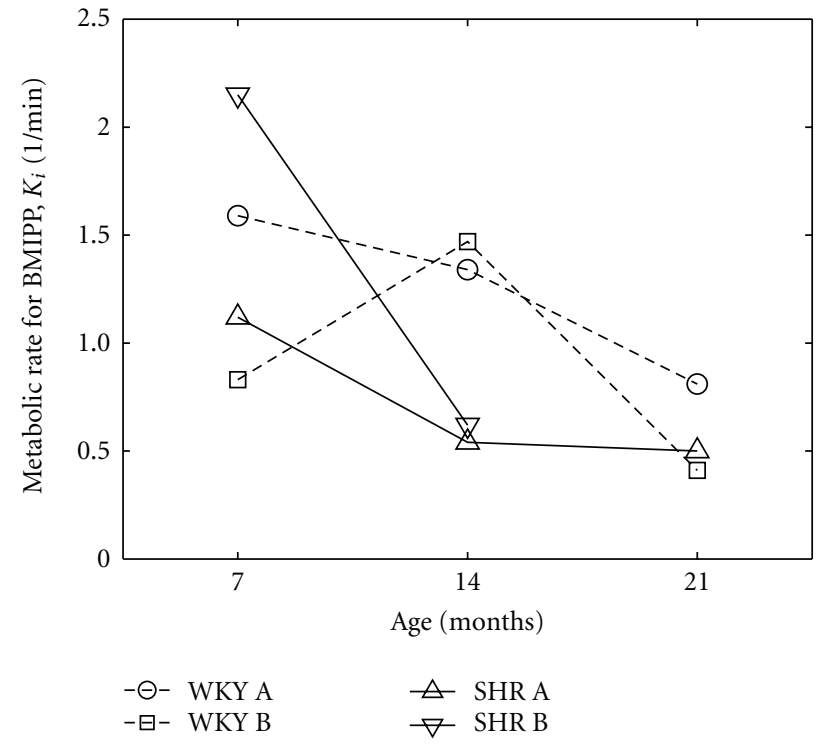

FIGURE 8: Metabolic rate of ${ }^{123} \mathrm{I}$-BMIPP in the myocardium as a function of age. SHR B died of congestive heart failure before 21 months.

spillover correction method compensated for partial volume effects and resulted in an increase of $72 \pm 45 \%$ in estimates of metabolic rates $K_{i}$, compared to estimates obtained without spillover correction. The results appear to indicate higher fatty metabolism in the control WKY rats as compared with SHRs. The fatty acid metabolic rate also decreases with age in both animal models. 
The review of Nohara [1] describes how BMIPP behaves in normal and diseased hearts at the cellular and molecular level. BMIPP is a 15-carbon chain with a methyl group in the $\beta$-position which inhibits oxidative metabolism and reduces myocardial washout kinetics. In the cell, $60 \%$ of BMIPP will be retained mostly in the triglyceride (TG) pool and has a longer retention than triglyceride, and $10 \%$ will be more or less metabolized to be washed out of the cell. Other than metabolizing to TG, the metabolites of ${ }^{123} \mathrm{I}$ BMIPP are mostly intermediate or final metabolites in the mitochondria, where ${ }^{123} \mathrm{I}-\mathrm{BMIPP}$ is metabolized to $\mathrm{p}-{ }^{123} \mathrm{I}-$ iodophenyl acetic acid (PIPA) by alpha-oxidation as the first step, followed by a beta-oxidation process to lactate. ${ }^{123}$ I-BMIPP reflects fatty acid uptake and the size of the lipid pool, and back diffusion of ${ }^{123}$ I-BMIPP and lactate production are good markers of ischemia. A 10-fold increase in the concentration of free fatty acid (FFA) in the blood will decrease ${ }^{123}$ I-BMIPP extraction by $25 \%$ and increase washout by $25 \%$, whereas a 2 -fold increase in the glucose concentration in the blood does not inhibit ${ }^{123}$ I-BMIPP uptake into the cell.

Lipid transport in the blood and in the cell is dependent upon it being bound to proteins or lipid proteins such as VLDL in the blood. Lipid is extracted into the cell by membrane proteins and is affected by albumin/FFA ratio in the blood. Higher lipid or FFA/albumin level results in greater uptake of lipid into the cell. Fatty acid binding protein (FABP) in the cytosole is one factor that regulates lipid flux. Lipid will be used either for oxidation, triglyceride or phospholipid formation. Carnitine palmityl transferese is the key enzyme for the entrance of lipid into mitochondria, and oxidative enzymes such as long chain acyl CoA dehydrogenase (LCAD) will determine lipid use into the TCA cycle. The eventual production of ATP in the mitochondria will limit the size of TG storage. The several lipid enzymes in the cell are regulated by nuclear genes that are individually activated by peroxisome proliferator activated receptors (PPARs). There are many endothelial proteins that are also involved in the storage and metabolism of lipid. The amount of medium chain acyl CoA dehydrogenase (MCAD) is closely related to the progression of heart failure. In the compensated phase of hypertrophy, m-RNA level of MCAD is reduced.

Even with species differences, our results (here for fatty acid metabolism, and $[6,7]$ for glucose metabolism) and other results obtained with the SHR model [38-40] corroborate studies in humans, which show that there are abnormalities in myocardial metabolic function manifested by reduced rates of fatty acid utilization and oxidation and an increase in glucose metabolism associated with hypertension-induced left ventricular hypertrophy (LVH) and idiopathic cardiomyopathy $[21,41]$. In a separate positron emission tomography (PET) study imaging ${ }^{18} \mathrm{~F}-$ fluorodeoxyglucose (FDG) with use of a microPET scanner, we observed that glucose metabolism in the SHR model was greater than that in the WKY control $[6,7]$, and that the glucose metabolism in both decreased with age. Present results differ from results imaging fatty acid utilization in patients with heart failure [13], where it was shown that myocardial fatty acid uptake in patients with heart failure was higher than that expected for the normal heart, whereas myocardial glucose uptake rates were lower. It may be that in the hypertrophied heart before heart failure there is greater dependency on glucose and with eventual heart failure there is a shift to fatty acid metabolism, and that this shift is an indication of impaired energy efficiency in the failing heart.

Our work also corresponds to work by Okizaki et al. [5]. Using dynamic SPECT imaging of ${ }^{123}$ I-BMIPP, they showed that fatty acid metabolism was higher in patients with hypertrophic cardiomyopathy. Using mathematical modeling, they showed that compartmental model rate parameters might be a sensitive predictor for early detection of hypertrophic cardiomyopathy and could be a useful index to evaluate its progression. A two-compartment model was used where the first compartment was a reversible compartment (cytoplasma) and the second compartment (triglyceride pool) was an irreversible compartment corresponding to the long retention of BMIPP if incorporated into the triglyceride pool. The compartmental analysis suggested that a fatty acid shift (rate of transport into the TG pool higher than normal and a decreased volume of distribution for the first compartment) from cytoplasm to the triglyceride pool occurs in hypertrophic cardiomyopathy, even in the early phase of the disease when no apparent change was observed upon visual interpretation by nuclear medicine physicians. Because of the computational demands, we were able to model only the first $90 \mathrm{~s}$ of the kinetics in the myocardium. We used a single, irreversible tissue compartment, and therefore were able to measure only the uptake of ${ }^{123} \mathrm{I}$ BMIPP, which we consider to be a measure of the metabolic rate of ${ }^{123} \mathrm{I}$-BMIPP. It is anticipated that an additional, reversible tissue compartment will be needed to account for washout of ${ }^{123}$ I-BMIPP from the myocardium over longer time scales.

${ }^{123}$ I-BMIPP imaging changes in the ischemic condition [1]. Disparity between ${ }^{123}$ I-BMIPP uptake in the hypertrophied myocardium and delayed ${ }^{201} \mathrm{Tl}$ redistribution images in rest/stress studies indicate that myocardial ischemia plays an important role in impaired fatty acid utilization and metabolism in hypertrophied myocardium [4]. Myocardial ischemia is present with hypertrophic cardiomyopathy for several reasons: small coronary artery disease, coronary artery spasms, left coronary artery compression, inadequate capillary density in relation to the increased myocardial mass, and impaired coronary flow reserve. Lipid metabolic regulation completely differs from normal in the very early phase of cardiac hypertrophy and with deteriorating heart failure, and metabolic switching from lipid to glucose will occur [1]. A slight reduction in flow will be reflected in an increase in glucose metabolism to a level 4-5 times the resting value, but the lipid metabolism will remain constant [1]. At less than $40 \%$ of resting flow, both lipid and glucose metabolisms decrease remarkably [1]. Extraction of lipids will decrease in cases of prolonged ischemia [1]. The myocardial accumulation of ${ }^{123}$ I-BMIPP is also related to ATP content, and thus may reflect pO2 levels [4]. Decreased myocardial ${ }^{123} \mathrm{I}$-BMIPP uptake in areas of stress-induced ischemia on ${ }^{201} \mathrm{Tl}$ imaging indicated that exercised-induced metabolic changes persisted even in the resting condition 
[4]. The possibility of an unknown hereditary fatty acid metabolic abnormality that could account for reduced ${ }^{123} \mathrm{I}$ BMIPP accumulation in hypertrophic cardiomyopathy has also been reported [4].

Our studies show a decrease in fatty acid metabolism in both the SHR and WKY rat with age. In one study in humans it was observed that myocardial fatty acid utilization (MFAU) and myocardial fatty acid oxidation (MFAO) declined with age while myocardial glucose utilization (MGU) did not change; thus, the proportional contribution of glucose used to overall substrate utilization relative to fatty acids was increased [42]. The decline in MFAO and MFAU with age may reflect a decline in mitochondrial long-chain fatty acid uptake [43], and alterations in mitochondrial lipid content, composition, and protein interactions, leading to significant membrane dysfunction [44] and effects of impaired myocardial vasodilator capacity [45]. The study in humans [42] appears to correlate with some studies in mouse and rat experimental models that show that the contribution of MFAO to overall myocardial substrate metabolism declines with age, and that the proportion of glucose metabolism to overall substrate metabolism increases [46]. However, one study in rats [47] appears to contradict these results showing that during the transition from adulthood to senescence, there is an increase in palmitate (fatty acid) oxidation and a decrease in lactate oxidation, and that this is associated with significant deterioration in cardiac function and efficiency. This paper suggests that the metabolic changes occur in parallel with hypertrophy. Thus, if hypertrophy is involved there could be an accompanied disproportionate enlargement in cell length causing an increase in the cell length-to-width ratio [48]. Cell loss, which increases with age [49], may also play an increasing role in the transition to heart failure by placing a greater workload on the remaining viable cells. The detrimental effect of increased fatty acid utilization observed in the senescent heart may be attributed to the fact that fatty acids are a less efficient fuel in terms of myocardial oxygen consumption (MVO2) [50].

Limitations of our present study are (1) small sample size (two WKY, two SHR), (2) animals were not fasted, (3) no blood pressure data, (4) no independent validation of the input function either from blood samples or from simulation, (5) data were not corrected for attenuation or scatter, and (6) only the first $90 \mathrm{~s}$ of the dynamic data were processed. Regarding fasting, it was felt that the animals had fairly similar free fatty acid, insulin, and glucose levels, which we verified in another study in which we sampled blood. Blood pressure is very difficult to obtain in these animals. Presently, simulations with known blood input functions are underway to determine the bias and variance that one would obtain with the experimental data. In future studies we plan to implement attenuation and scatter correction using the transmission source on the GE Hawkeye SPECT/CT system. We have performed simulations that showed improvement in quantitation using scatter and attenuation correction [35]. In a rat it improves quantitation by $10-15 \%$. Future work will also include addressing computational issues associated with reconstructing a 4-D dynamic image from the entire $60 \mathrm{~min}$ of projection data.
Our injected dose of $4 \mathrm{mCi}(150 \mathrm{MBq})$ is high when compared with dose/weight ratios of what one would give a human. The injected dose was based on our past experience $[8,9]$ as to what we anticipated the photon counting statistics needed to be to perform the data analysis. Our statistical uncertainties appear to be reasonable. Nonetheless, this still needs to be evaluated in an extensive study of statistical precision based on injected dose in rats. We have not found any harmful effects to the animals using this dose; however, an extensive radiobiology study has not been performed.

The work presented here relates primarily to the development of technology for dynamic pinhole SPECT imaging of small animals. The results are the first to report on being able to measure fatty acid metabolic rate in rats with use of a clinical pinhole SPECT system with slowly-rotating detectors. From a technical standpoint, this measurement was made possible in part because of the slow (10-30 s) injection of ${ }^{123}$ I-BMIPP, which allowed good angular sampling of the projections of the blood input function. The slow injection was also necessary, so as not to overwhelm the rat's circulatory system with the additional volume $(\sim 1 \mathrm{~mL})$ of the injectate. For faster injections of smaller volumes of injectate, an imaging system with stationary detectors, such as the U-SPECT II preclinical scanner [51], is better-suited for tomographic imaging of the resulting faster kinetics. The USPECT II also has the advantage of having a collimator with $751-\mathrm{mm}$ pinholes for use with imaging rats, which provides about 13 times the sensitivity of our two-pinhole system and the ability to resolve $0.8-\mathrm{mm}$ "cold" rods in a radioactive background [51]. Sensitivity and spatial resolution of our system could also be improved by judiciously increasing the number of and decreasing the size of the pinholes.

Our methods can also be applied to other imaging modalities, such as dynamic PET. We are presently studying fatty acid metabolism as a function of age in SHR and WKY rats using microPET imaging of the fatty acid analog, $14(R, S)$ - $\left[{ }^{18} \mathrm{~F}\right]$ fluoro-6-thia-heptadecanoic acid $\left({ }^{18} \mathrm{~F}-\mathrm{FTHA}\right)$ [52]. Combining new dynamic and conventional clinical imaging protocols with improved descriptions of the heart (physiological, mechanical, and biochemical) will allow better specification of the heart's properties. This, in turn, will facilitate the study of how these properties are affected by molecular changes in the heart caused by disease.

\section{Disclaimer}

This document was prepared as an account of work sponsored by the United States Government. While this document is believed to contain correct information, neither the United States Government nor any agency thereof, nor The Regents of the University of California, nor any of their employees, makes any warranty, express or implied, or assumes any legal responsibility for the accuracy, completeness, or usefulness of any information, apparatus, product, or process disclosed, or represents that its use would not infringe privately owned rights. Reference herein to any specific commercial product, process, or service by its trade name, trademark, manufacturer, or otherwise, does not necessarily constitute or imply its endorsement, recommendation, or favoring by 
the United States Government or any agency thereof, or The Regents of the University of California. The views and opinions of authors expressed herein do not necessarily state or reflect those of the United States Government or any agency thereof or The Regents of the University of California.

\section{Acknowledgment}

This paper was supported by the National Institutes of Health of the U. S. Department of Health and Human Services under Grants R01-EB00121, R01-EB007219, R01HL50663, and R01-HL71253; and by the Director, Office of Science, Office of Biological and Environmental Research of the U. S. Department of Energy under contract DE-AC0205CH11231.

\section{References}

[1] R. Nohara, "Lipid metabolism in the heart: contribution of BMIPP to the diseased heart," Annals of Nuclear Medicine, vol. 15 , no. 5, pp. 403-409, 2001.

[2] L. H. Opie, The Heart: Physiology and Metabolism, Raven Press, New York, NY, USA, 2nd edition, 1991.

[3] J. R. Neely and H. E. Morgan, "Relationship between carbohydrate and lipid metabolism and the energy balance of heart muscle," Annual Review of Physiology, vol. 36, pp. 413-459, 1974.

[4] S. Matsuo, Y. Nakamura, M. Takahashi, K. Mitsunami, and M. Kinoshita, "Myocardial metabolic abnormalities in hypertrophic cardiomyopathy assessed by iodine-123-labeled beta-methyl-branched fatty acid myocardial scintigraphy and its relation to exercise-induced ischemia," Japanese Circulation Journal, vol. 62, no. 3, pp. 167-172, 1998.

[5] A. Okizaki, N. Shuke, J. Sato et al., "A compartment model analysis for investigation of myocardial fatty acid metabolism in patients with hypertrophic cardiomyopathy," Nuclear Medicine Communications, vol. 28, no. 9, pp. 726-735, 2007.

[6] G. T. Gullberg, R. H. Huesman, J. Qi, B. W. Reutter, A. Sitek, and Y. Yang, "Evaluation of cardiac hypertrophy in spontaneously hypertensive rats using metabolic rate of glucose estimated from dynamic microPET image data," Molecular Imaging, vol. 3, no. 3, p. 225, 2004.

[7] G. T. Gullberg, R. H. Huesman, B. W. Reutter et al., "A study of changes in deformation and metabolism in the left ventricle as a function of hypertrophy in spontaneous hypertensive rats using microPET technology," Journal of Nuclear Cardiology, vol. 12, no. 2, p. S74, 2005.

[8] J. Hu, A. Sitek, B. W. Reutter, R. H. Huesman, and G. T. Gullberg, "A new approach of dynamic pinhole SPECT imaging for evaluation of sympathetic nervous system function in animal models of cardiac hypertrophy," in Proceedings of the IEEE Nuclear Science Symposium and Medical Imaging Conference Record, B. Yu, Ed., pp. 2542-2546, 2005.

[9] J. Hu, A. Sitek, B. W. Reutter, R. H. Huesman, and G. T. Gullberg, "Dynamic molecular imaging of cardiac innervation using a dual head pinhole SPECT system," Tech. Rep. LBNL60008, Lawrence Berkeley National Laboratory, 2006.

[10] A. B. Hwang, B. W. Reutter, R. H. Huesman et al., "High resolution simultaneous I-125 and Tc-99 $\mathrm{m}$ cardiac imaging of a rat using a conventional SPECT system with pin-hole collimators," Journal of Nuclear Medicine, vol. 45, no. 5, pp. 422P-423P, 2004.
[11] A. B. Hwang, B. L. Franc, G. T. Gullberg, and B. H. Hasegawa, "Assessment of the sources of error affecting the quantitative accuracy of SPECT imaging in small animals," Physics in Medicine and Biology, vol. 53, no. 9, pp. 2233-2252, 2008.

[12] T. Hajri, A. Ibrahimi, C. T. Coburn et al., "Defective fatty acid uptake in the spontaneously hypertensive rat is a primary determinant of altered glucose metabolism, hyperinsulinemia, and myocardial hypertrophy," Journal of Biological Chemistry, vol. 276, no. 26, pp. 23661-23666, 2001.

[13] M. Taylor, T. R. Wallhaus, T. R. Degrado et al., "An evaluation of myocardial fatty acid and glucose uptake using PET with [18F]fluoro-6-thia-heptadecanoic acid and [18F]FDG in patients with congestive heart failure," Journal of Nuclear Medicine, vol. 42, no. 1, pp. 55-62, 2001.

[14] O. D. Mjos, "Effect of free fatty acids on myocardial function and oxygen consumption in intact dogs," Journal of Clinical Investigation, vol. 50, no. 7, pp. 1386-1389, 1971.

[15] H. Taegtmeyer, "Energy metabolism of the heart: from basic concepts to clinical applications," Current Problems in Cardiology, vol. 19, no. 2, pp. 59-113, 1994.

[16] F. C. Visser, "Imaging of cardiac metabolism using radiolabelled glucose, fatty acids and acetate," Coronary Artery Disease, vol. 12, supplement 1, pp. S12-S18, 2001.

[17] S. P. Bishop and R. A. Altschuld, "Increased glycolytic metabolism in cardiac hypertrophy and congestive failure," The American Journal of Physiology, vol. 218, no. 1, pp. 153159, 1970.

[18] M. E. Chiste and R. L. Rodgers, "Altered glucose and fatty acid oxidation in hearts of the spontaneously hypertensive rat," Journal of Molecular and Cellular Cardiology, vol. 26, no. 10, pp. 1371-1375, 1994.

[19] K. Kataoka, R. Nohara, R. Hosokawa et al., "Myocardial lipid metabolism in compensated and advanced stages of heart failure: evaluation by canine pacing model with BMIPP," Journal of Nuclear Medicine, vol. 42, no. 1, pp. 124-129, 2001.

[20] F. F. Knapp Jr. and J. Kropp, "BMIPP-design and development," International Journal of Cardiac Imaging, vol. 15, no. 1, pp. 1-9, 1999.

[21] V. G. Dávila-Román, G. Vedala, P. Herrero et al., “Altered myocardial fatty acid and glucose metabolism in idiopathic dilated cardiomyopathy," Journal of the American College of Cardiology, vol. 40, no. 2, pp. 271-277, 2002.

[22] F. A. Recchia, P. I. McConnell, R. D. Bernstein, T. R. Vogel, X. $\mathrm{Xu}$, and T. H. Hintze, "Reduced nitric oxide production and altered myocardial metabolism during the decompensation of pacing-induced heart failure in the conscious dog," Circulation Research, vol. 83, no. 10, pp. 969-979, 1998.

[23] M. N. Sack, T. A. Rader, S. Park, J. Bastin, S. A. McCune, and D. P. Kelly, "Fatty acid oxidation enzyme gene expression is downregulated in the failing heart," Circulation, vol. 94, no. 11, pp. 2837-2842, 1996.

[24] C. Brunold, Z. El Alaoui-Talibi, M. Moravec, and J. Moravec, "Palmitate oxidation by the mitochondria from volumeoverloaded rat hearts," Molecular and Cellular Biochemistry, vol. 180, no. 1-2, pp. 117-128, 1998.

[25] J. J. Lehman and D. P. Kelly, "Gene regulatory mechanisms governing energy metabolism during cardiac hypertrophic growth," Heart Failure Reviews, vol. 7, no. 2, pp. 175-185, 2002.

[26] M. N. Sack and D. P. Kelly, “The energy substrate switch during development of heart failure: gene regulatory mechanisms (Review)," International Journal of Molecular Medicine, vol. 1, no. 1, pp. 17-24, 1998. 
[27] B. W. Reutter, G. T. Gullberg, R. Boutchko et al., "Fully 4D dynamic cardiac SPECT image reconstruction using spatiotemporal B-spline voxelization," in Proceedings of the IEEE Nuclear Science Symposium and Medical Imaging Conference Record, B. Yu, Ed., pp. 4217-4221, 2007.

[28] B. W. Reutter, R. Boutchko, R. H. Boutchko et al., "Dynamic pinhole SPECT imaging and compartmental modeling of fatty acid metabolism in the rat heart," in Proceedings of the IEEE Nuclear Science Symposium and Medical Imaging Conference Record, P. Sellin, Ed., pp. 4478-4481, 2008.

[29] B. W. Reutter, R. Boutchko, R. H. Huesman, A. C. Sauve, and G. T. Gullberg, "Penalized least-squares dynamic pinhole SPECT image reconstruction using a smooth 4-D image prior and multiresolution spatiotemporal B-splines," in Proceedings of the 10th International Conference on Fully ThreeDimensional Image Reconstruction in Radiology and Nuclear Medicine, B. M. W. Tsui and Z. Chen, Eds., pp. 110-113, 2009.

[30] B. F. Hutton and A. Osiecki, "Correction of partial volume effects in myocardial SPECT," Journal of Nuclear Cardiology, vol. 5, no. 4, pp. 402-413, 1998.

[31] P. H. Pretorius and M. A. King, "Diminishing the impact of the partial volume effect in cardiac SPECT perfusion imaging," Medical Physics, vol. 36, no. 1, pp. 105-115, 2009.

[32] R. H. Huesman, B. L. Knittel, B. M. Mazoyer et al., "Notes on RFIT: a program for fitting compartmental models to regionof-interest dynamic emission tomography data," Tech. Rep. LBL-37621, Lawrence Berkeley National Laboratory, 1995.

[33] R. J. Jaszczak, J. Li, H. Wang, M. R. Zalutsky, and R. E. Coleman, "Pinhole collimation for ultra-high-resolution, small-field-of-view SPECT," Physics in Medicine and Biology, vol. 39, no. 3, pp. 425-437, 1994.

[34] A. Sauve, W. Choong, R. Boutchko et al., "Quantitative imaging of cardiovascular function with pinhole SPECT in mice and rats-effects of attenuation and scatter," Journal of Nuclear Medicine, vol. 48, no. 5, pp. 424P-425P, 2007.

[35] A. C. Sauve, B. W. Reutter, R. Boutchko et al., "Multi-slice SPECT attenuation and scatter correction using Monte Carlo simulated system matrices," in Proceedings of the IEEE Nuclear Science Symposium and Medical Imaging Conference Record, P. Sellin, Ed., pp. 4273-4276, 2008.

[36] B. W. Reutter, G. T. Gullberg, and R. H. Huesman, "Kinetic parameter estimation from attenuated SPECT projection measurements," IEEE Transactions on Nuclear Science, vol. 45, no. 6, pp. 3007-3013, 1998.

[37] B. W. Reutter, S. Oh, G. T. Gullberg, and R. H. Huesman, "Improved quantitation of dynamic SPECT via fully 4-D joint estimation of compartmental models and blood input function directly from projections," in Proceedings of the IEEE Nuclear Science Symposium and Medical Imaging Conference Record, B. Yu, Ed., pp. 2337-2341, 2005.

[38] O. H. L. Bing, C. H. Conrad, M. O. Boluyt, K. G. Robinson, and W. W. Brooks, "Studies of prevention, treatment and mechanisms of heart failure in the aging spontaneously hypertensive rat," Heart Failure Reviews, vol. 7, no. 1, pp. 7188, 2002.

[39] O. H. Cingolani, X.-P. Yang, M. A. Cavasin, and O. A. Carretero, "Increased systolic performance with diastolic dysfunction in adult spontaneously hypertensive rats," Hypertension, vol. 41, no. 2, pp. 249-254, 2003.

[40] J. E. Jalil, C. W. Doering, J. S. Janicki, R. Pick, S. G. Shroff, and K. T. Weber, "Fibrillar collagen and myocardial stiffness in the intact hypertrophied rat left ventricle," Circulation Research, vol. 64, no. 6, pp. 1041-1050, 1989.
[41] L. de las Fuentes, P. F. Soto, B. P. Cupps et al., "Hypertensive left ventricular hypertrophy is associated with abnormal myocardial fatty acid metabolism and myocardial efficiency," Journal of Nuclear Cardiology, vol. 13, no. 3, pp. 369-377, 2006.

[42] A. M. Kates, P. Herrero, C. Dence et al., "Impact of aging on substrate metabolism by the human heart," Journal of the American College of Cardiology, vol. 41, no. 2, pp. 293-299, 2003.

[43] J. A. Odiet, M. E. T. I. Boerrigter, and J. Y. Wei, "Carnitine palmitoyl transferase-I activity in the aging mouse heart," Mechanisms of Ageing and Development, vol. 79, no. 2-3, pp. 127-136, 1995.

[44] G. Paradies, F. M. Ruggiero, G. Petrosillo, M. N. Gadaleta, and E. Quagliariello, "The effect of aging and acetyl-L-carnitine on the function and on the lipid composition of rat heart mitochondria," Annals of the New York Academy of Sciences, vol. 717, pp. 233-243, 1994.

[45] A. Chauhan, R. S. More, P. A. Mullins, G. Taylor, M. C. Petch, and P. M. Schofield, "Aging-associated endothelial dysfunction in humans is reversed by L-arginine," Journal of the American College of Cardiology, vol. 28, no. 7, pp. 1796-1804, 1996.

[46] J. B. McMillin, G. E. Taffet, H. Taegtmeyer, E. K. Hudson, and C. A. Tate, "Mitochondrial metabolism and substrate competition in the aging Fischer rat heart," Cardiovascular Research, vol. 27, no. 12, pp. 2222-2228, 1993.

[47] J. Sample, J. G. F. Cleland, and A.-M. L. Seymour, "Metabolic remodeling in the aging heart," Journal of Molecular and Cellular Cardiology, vol. 40, no. 1, pp. 56-63, 2006.

[48] A. M. Gerdes, T. Onodera, X. Wang, and S. A. McCune, "Myocyte remodeling during the progression to failure in rats with hypertension," Hypertension, vol. 28, no. 4, pp. 609-614, 1996.

[49] G. Olivetti, M. Melissari, J. M. Capasso, and P. Anversa, "Cardiomyopathy of the aging human heart. Myocyte loss and reactive cellular hypertrophy," Circulation Research, vol. 68, no. 6, pp. 1560-1568, 1991.

[50] C. S. Apstein, "Increased glycolytic substrate protection improves ischemic cardiac dysfunction and reduces injury," American Heart Journal, vol. 139, no. 2, part 3, pp. S107-S114, 2000.

[51] F. D. Van Have, B. Vastenhouw, R. M. Ramakers et al., "U-SPECT-II: an ultra-high-resolution device for molecular small-animal imaging," Journal of Nuclear Medicine, vol. 50, no. 4, pp. 599-605, 2009.

[52] H. Wahnishe, J. O’Neil, M. Janabi et al., "Cardiac metabolism as a function of cardiac hypertrophy using microPET/CT imaging of [F-18]FTHA and [F-18]FDG," Journal of Nuclear Medicine, vol. 51, supplement 2, p. 324, 2010. 


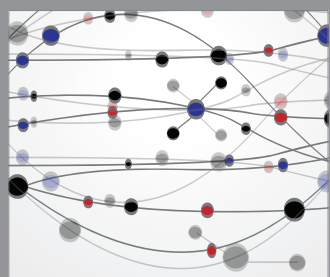

The Scientific World Journal
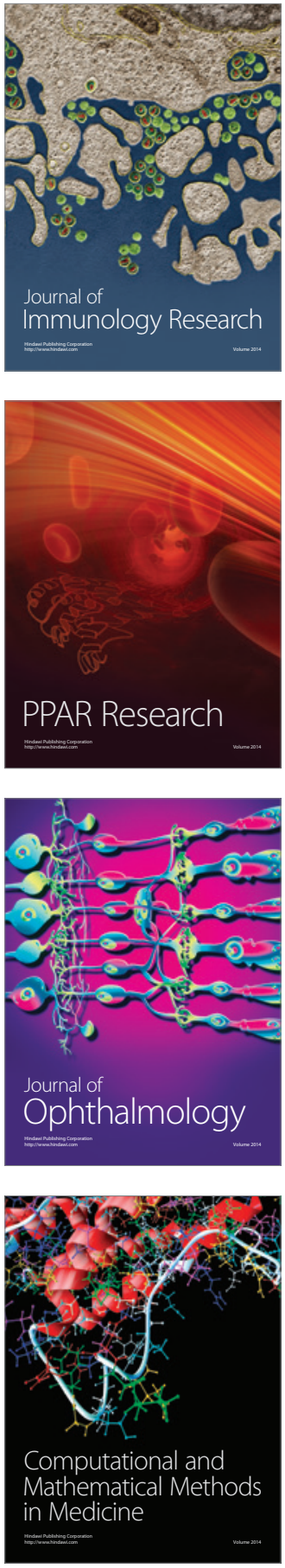

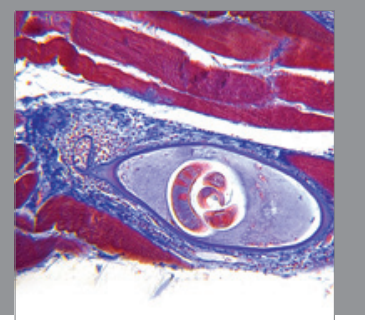

Gastroenterology

Research and Practice
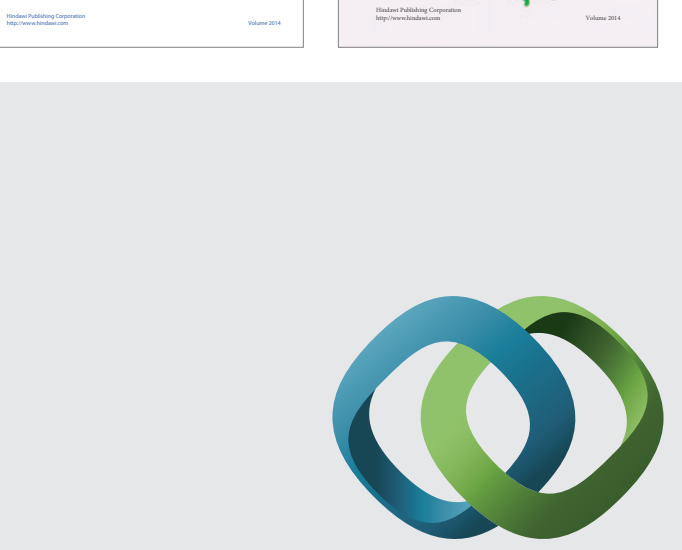

\section{Hindawi}

Submit your manuscripts at

http://www.hindawi.com
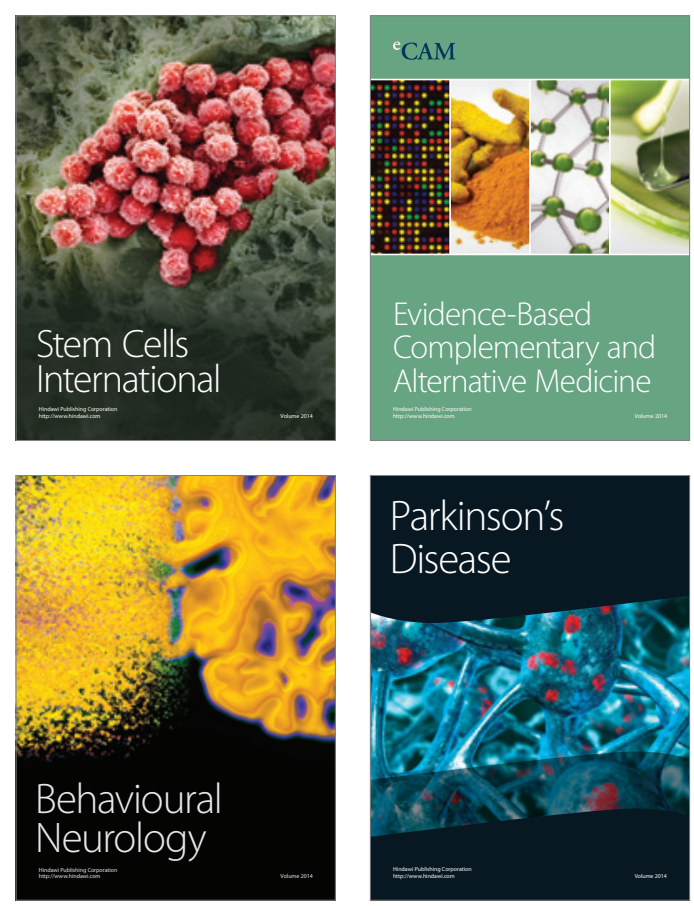

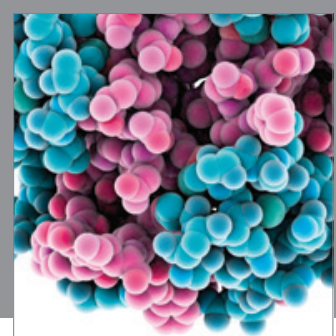

Journal of
Diabetes Research

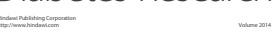

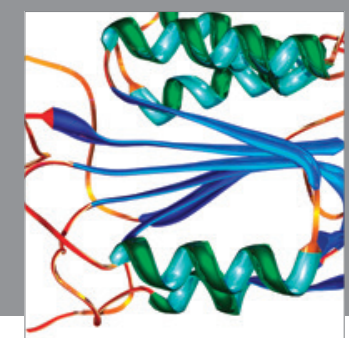

Disease Markers
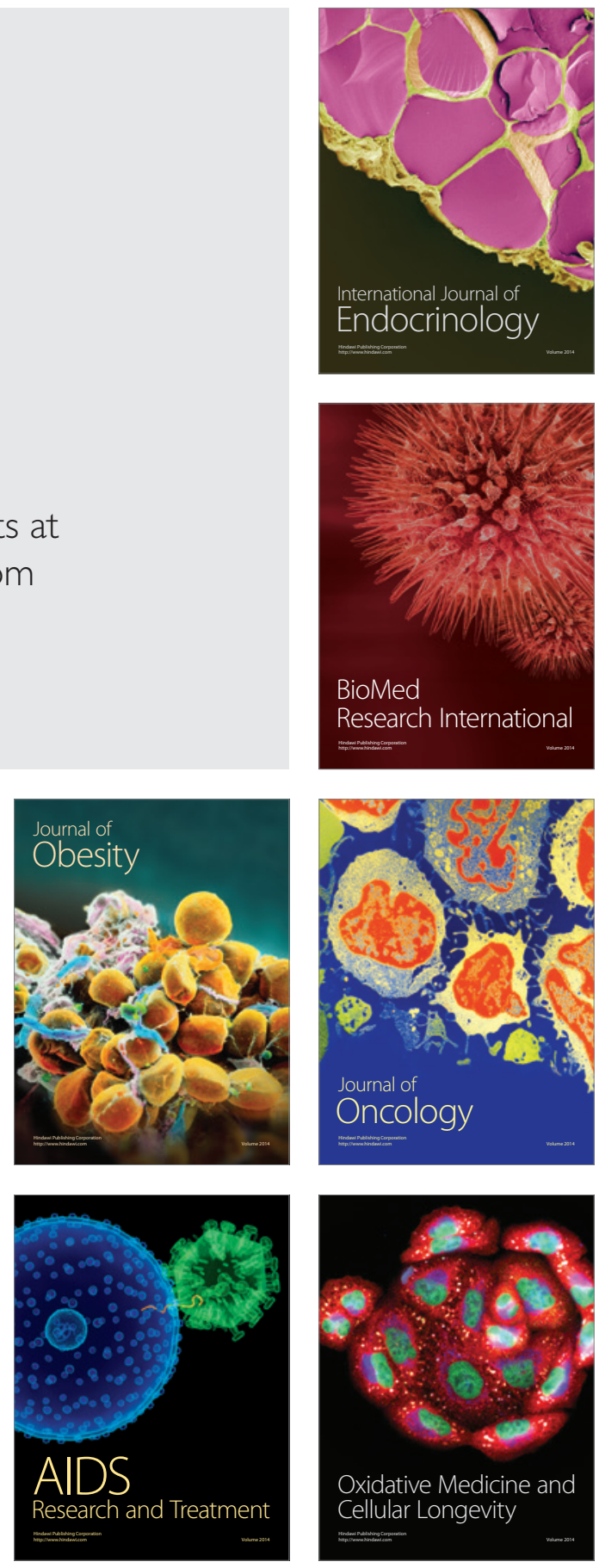